\title{
A latent consolidation phase in auditory identification learning: Time in the awake state is sufficient
}

\author{
Daphne Ari-Even Roth, ${ }^{1,4}$ Liat Kishon-Rabin, ${ }^{1}$ Minka Hildesheimer, ${ }^{1}$ and Avi Karni ${ }^{2,3}$ \\ ${ }^{1}$ Department of Communication Disorders, Sackler Faculty of Medicine, Tel Aviv University, Tel Hashomer 52621, Israel; ${ }^{2}$ The \\ Brain-Behavior Research Center, Faculties of Science \& Education, University of Haifa, Mt. Carmel 31905, Israel; ${ }^{3}$ Departments of \\ Neurology and Diagnostic Radiology, The Chaim Sheba Medical Center, Tel Hashomer 52621, Israel
}

\begin{abstract}
Large gains in performance, evolving hours after practice has terminated, were reported in a number of visual and some motor learning tasks, as well as recently in an auditory nonverbal discrimination task. It was proposed that these gains reflect a latent phase of experience-triggered memory consolidation in human skill learning. It is not clear, however, whether and when delayed gains in performance evolve following training in an auditory verbal identification task. Here we show that normal-hearing young adults trained to identify consonant-vowel stimuli in increasing levels of background noise showed significant, robust, delayed gains in performance that became effective not earlier than $4 \mathrm{~h}$ post-training, with most participants improving at more than $6 \mathrm{~h}$ post-training. These gains were retained for over 6 mo. Moreover, although it has been recently argued that time including sleep, rather than time per se, is necessary for the evolution of delayed gains in human perceptual learning, our results show that $12 \mathrm{~h}$ post-training in the waking state were as effective as $12 \mathrm{~h}$, including no less than $6 \mathrm{~h}$ night's sleep. Altogether, the results indicate, for the first time, the existence of a latent, hours-long, consolidation phase in a human auditory verbal learning task, which occurs even during the awake state.
\end{abstract}

A leading notion in current memory research is that the acquisition of procedural (how to) memory, specifically skill learning, is a multistep process continuing beyond the actual training experience (Karni and Sagi 1993; Korman et al. 2003; Maquet et al. 2003a). One characteristic of adult skill learning is that incremental, time-dependent gains in performance evolve, in a latent manner, hours after the termination of practice (see Karni and Sagi 1993; Karni et al. 1994; Stickgold 1998; Hauptmann and Karni 2002; Maquet et al. 2003a). There are several indications that during this latent phase, both quantitative and qualitative changes occur in the knowledge gained during the training experience (Karni and Sagi 1993; Brashers-Krug et al. 1996; Hikosaka et al. 1999; Korman et al. 2003; Walker et al. 2003). The evolution of delayed gains in performance was proposed to reflect the properties of a limited set of basic mechanisms of neuronal plasticity throughout the adult cortex (Karni and Sagi 1993; Karni 1996; Karni and Bertini 1997).

Delayed gains in performance were clearly shown in different versions of a visual texture discrimination learning task (see Karni and Sagi 1991, 1993; Karni et al. 1994; Stickgold 1998; Gais et al. 2000; Stickgold et al. 2000a,b), in a visual enumeration task (Hauptmann and Karni 2002), in a motor sequence learning paradigm (Fischer et al. 2002; Walker et al. 2002; Korman et al. 2003) and in visuo-motor tasks (e.g., Maquet et al. 2000, 2003b). Recently, delayed gains were reported in an auditory nonverbal pitch memory task (Gaab et al. 2004), but only if sleep was allowed. Others, however, investigating the role of sleep in complex nonverbal auditory learning tasks found no delayed gains, compared with immediate post-training performance, either with or without sleep (Atienza et al. 2004; Gottselig et al. 2004).

\footnotetext{
${ }^{4}$ Corresponding author.

Email rothar@inter.net.il; fax 972-3-5352868

Article and publication are at http://www.learnmem.org/cgi/doi/10.1101/ 87505 .
}

There is growing evidence that sleep may play an important role in the evolution of delayed gains in performance (for review, see Maquet et al. 2003a). While it has been established that time including sleep contributes to the evolution of delayed gains in visual (see Karni et al. 1994; Stickgold 1998), and perhaps more so in motor (see Walker et al. 2002) skill learning, an important unresolved issue is whether time including sleep, rather than time per se, is a prerequisite or only a facilitating factor for the evolution of these gains (Karni and Sagi 1993; Karni et al. 1994; Maquet et al. 2000, 2003b; Stickgold et al. 2000a,b; Peigneux et al. 2001; Fischer et al. 2002; Walker et al. 2002; Korman et al. 2003).

The aim of the current study was to investigate whether delayed gains in performance significantly contribute to human learning in a verbal auditory identification task, and to study the time-course of evolution and retention of such gains. The results show that (1) training resulted in robust, long-lasting, timedependent delayed gains in performance in normal hearing young adults; and (2) time (on the order of a few hours) in the awake state was sufficient for the evolution of robust delayed gains in auditory performance.

\section{Results}

The design of the study (experiments 1 and 2) is shown in Figure 1. A single intensive training session on a consonant-vowel (CV) syllables-in-noise identification task resulted in significant timedependent latent gains in performance in 44 of 48 participants at $24 \mathrm{~h}$ post-training. No significant gains in performance occurred in the nine of 10 subjects, who were tested within the first $5 \mathrm{~h}$ post-training, with some participants showing a decrease in performance (Fig. 2) (mean improvement $=-0.61 \% \pm 4.57$; $\mathrm{d}^{\prime}=1.8 \pm 0.23$ and $1.73 \pm 0.21$ at training and $1-5 \mathrm{~h}$ posttraining, respectively). However, eight of 10 participants who were tested at 6-12 $\mathrm{h}$ post-training (time spent in the awake state) 
A

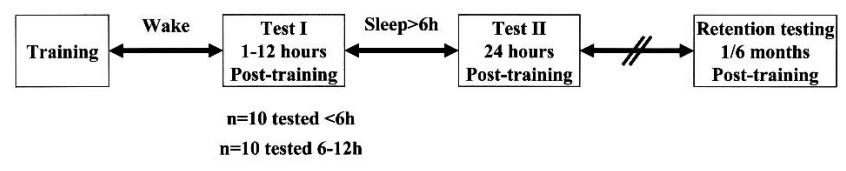

B

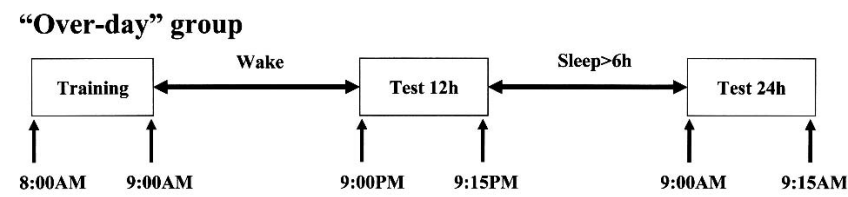

“Over-night" group

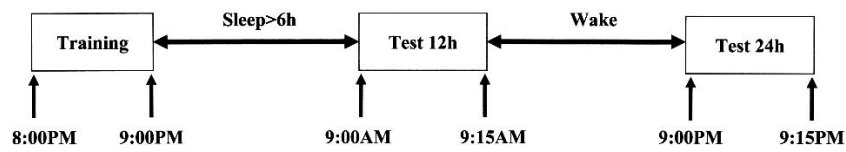

Figure 1. CV-syllables-in-noise identification task: training and testing protocol. Training in both experiments was identical. $(A)$ Experiment 1 protocol including long-term retention at 1 or 6 mo post-training. (B) Experiment 2 protocol (see Design and procedure).

showed significant gains in performance (ANOVA, $F_{(1,9)}=157.66 ; P<0.001$, mean improvement $=5.07 \% \pm 3.04$; $\mathrm{d}^{\prime}=1.91 \pm 0.22$ and $2.39 \pm 0.25$ at training and $6-12 \mathrm{~h}$ posttraining, respectively) (Fig. 2). All 20 of the above participants showed significant robust gains in performance on retesting at 24 $\mathrm{h}$ post-training (Fig. 2). By that time, both groups showed similar gains in performance (mean improvement $=9.24 \% \pm 3.22$ and $9.19 \% \pm 1.93 ;$ and $\mathrm{d}^{\prime}=2.55 \pm 0.38$ and $2.86 \pm 0.34$ for the $1-5$ and $6-12 \mathrm{~h}$ post-training groups, respectively). At $48 \mathrm{~h}$ posttraining, no additional gains in performance were observed compared with $24 \mathrm{~h}$ post-training $(8.46 \% \pm 3.19$ and $8.17 \% \pm 1.61$, respectively, $P=\mathrm{NS}$ ).

The possibility that gains in performance observed $24 \mathrm{~h}$ post-training were influenced by the experience gained during the first post-training test (at 1-12 $\mathrm{h}$ post-training) was excluded as no significant differences in the gains were observed at $24 \mathrm{~h}$ post-training between the control group participants-who were tested only once (at $24 \mathrm{~h}$ post-training) —and those obtained at $24 \mathrm{~h}$ post-training in the participants who were tested also at $1-12 \mathrm{~h}$ post-training (mean improvement $=9.33 \% \pm 1.4$ and $9.45 \% \pm 2.08$; and $\mathrm{d}^{\prime}=0.84 \pm 0.32$ and $0.92 \pm 0.22$ in the control and experiment 1 groups, respectively, $P=\mathrm{NS}$ ).

Performance gains attained by $24 \mathrm{~h}$ post-training were fully maintained in all (nine of nine) individuals that were retested at 1 mo post-training (mean performance $=90.22 \% \pm 3.95$ and $88.56 \% \pm 1.96 ; \mathrm{d}^{\prime}=2.77 \pm 0.49$ and $2.59 \pm 0.27$ at $24 \mathrm{~h}$ and 1 mo post-training, respectively), and in all (six of six) individuals tested at 6 mo post-training, with no training in the interval (mean performance $=89.5 \% \pm 2.83$ and $90.58 \% \pm 3.46$; $\mathrm{d}^{\prime}=2.6 \pm 0.3$ and $2.82 \pm 0.33$ at $24 \mathrm{~h}$ and 6 mo post-training, respectively) (Fig. 2).

In experiment 2, two groups of participants were tested for a possible differential effect of equal intervals of time posttraining during daytime (in wakefulness) or overnight (including a normal sleep of $>6 \mathrm{~h}$ duration) on performance at different time points post-training. No significant differences in the initial performance (at each signal-to-noise ratio [SNR]) were observed between the two groups, reducing the likelihood of circadian effects on performance (Fig. 3A). Significant gains in performance, of similar magnitude, were obtained at $12 \mathrm{~h}$ post-training in both groups. The over-day group improved by $6.17 \% \pm 3.52$ (ANOVA,
$F_{(1,8)}=27.66, \mathrm{p}=0.001 ; \mathrm{d}^{\prime}=1.74 \pm 0.25$ and $2.31 \pm 0.29$ at training and $12 \mathrm{~h}$ post-training, respectively) and the overnight group by $5.72 \% \pm 3.12$ (ANOVA, $F_{(1,8)}=30.2, P=0.001$; $\mathrm{d}^{\prime}=1.77 \pm 0.42$ and $2.3 \pm 0.33$ at training and $12 \mathrm{~h}$ posttraining, respectively). In both groups, additional gains in performance occurred between the 12- and 24-h post-training measurements. Some individuals, in both training groups, showed large gains by $12 \mathrm{~h}$ post-training with no additional gains thereafter, while others showed little improvement at $12 \mathrm{~h}$ posttraining, but considerably improved after additional $12 \mathrm{~h}$ (i.e., by $24 \mathrm{~h}$ post-training) (Fig. 3B).

There were no significant differences between the means of the two groups at 12 and $24 \mathrm{~h}$ post-training, with an interval of $12 \mathrm{~h}$ in the waking state as effective as equal time overnight including at least $6 \mathrm{~h}$ of sleep (mean performance at $12 \mathrm{~h}$ posttraining $=86.44 \% \pm 2.78$ and $86.06 \% \pm 3.49, \mathrm{~d}^{\prime}=2.31 \pm 0.29$ and $2.30 \pm 0.33$ for the over-day and overnight groups, respectively (ANOVA, $\left.F_{(1,16)}=0.07, P=\mathrm{NS}\right)$; mean performance at $24 \mathrm{~h}$ post-training $=88.5 \% \pm 3.21$ and $88.06 \% \pm 3.17$, $\mathrm{d}^{\prime}=2.47 \pm 0.36$ and $2.59 \pm 0.45$ for the over-day and overnight groups, respectively (ANOVA, $F_{(1,16)}=0.09, P=$ NS). (Fig. 3B).

\section{Discussion}

Our results provide evidence for a latent, hours-long, posttraining phase of improvement in an auditory verbal identification task in normal hearing young adults. A single intensive training session on an auditory CV-syllables-in-noise identification task triggered large, significant gains in performance that emerged 4-6 $\mathrm{h}$ after the termination of the training experience. Moreover, the results of both our experiments suggest that the evolution of delayed gains in this task did not necessarily depend on sleep and that time in the waking state was sufficient. We propose that these time-dependent gains in performance reflect a memory consolidation phase (Karni and Sagi 1993; Karni and Bertini 1997; Stickgold et al. 2002; Maquet et al. 2003a) in human auditory learning. The finding that the gains in performance were retained over a 6-mo interval supports the notion that both the immediate and the delayed effects of training were consolidated into long-term memory (Karni and Sagi 1993).

While robust training effects in the auditory modality of human adults are well recognized (see Watson 1980; Demany 1985; Tremblay et al. 1998), the possibility that these improve-

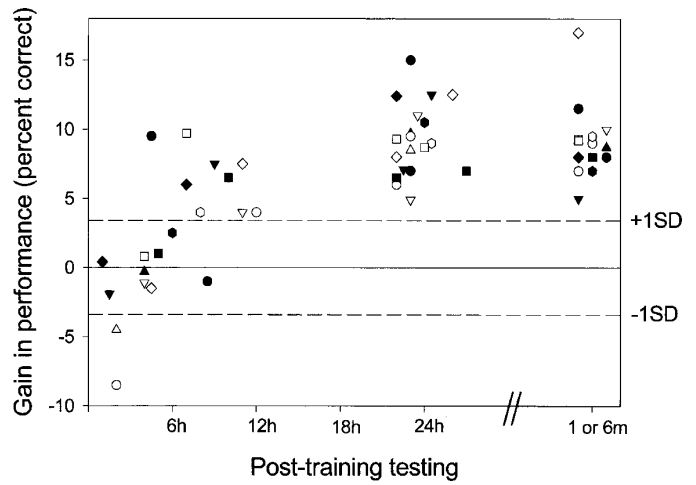

Figure 2. Individual gains in performance as a function of time posttraining (1-12 h, $24 \mathrm{~h}$, and 1 or 6 mo post-training). Gains were computed as the difference between identification scores (percentage correct) at different time points and the performance attained during the training session. The horizontal dashed lines represent the median of the standard deviations of all trained subjects at the level of $80 \%$ accuracy as attained during the training session. The gap in the time interval between $12-24 \mathrm{~h}$ post-training stands for nighttime including sleep of at least $6 \mathrm{~h}$. 
A

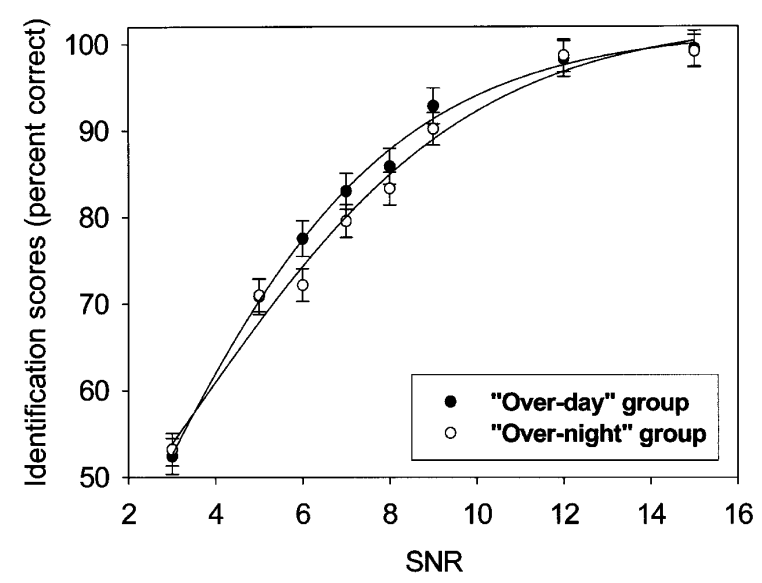

B

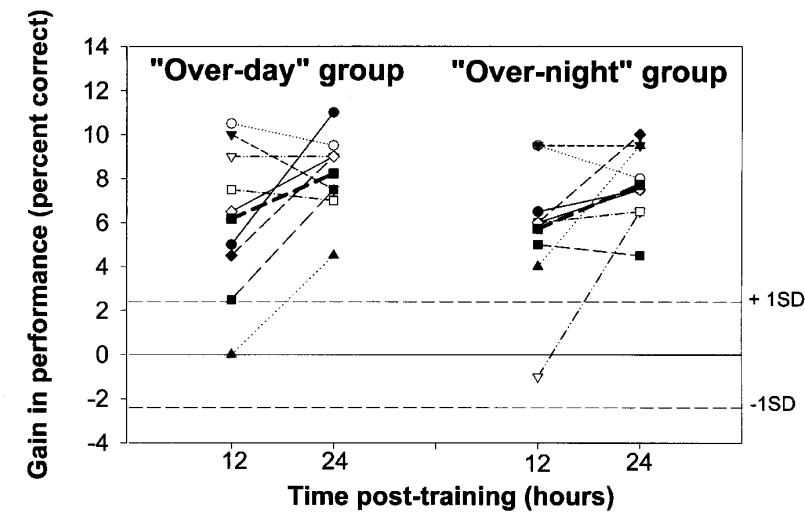

Figure 3. Performance of over-day and overnight groups. (A) Psychometric curves of performance (group mean \pm SEM) at training for the two groups. (B) Individual gains in performance (percentage correct) after training for the over-day and overnight groups, at $12 \mathrm{~h}$ and $24 \mathrm{~h}$ post-training. Bold squares and solid line show group mean performance. The horizontal dashed lines represent the median of the standard deviations of all trained subjects at the level of $80 \%$ accuracy as attained during the training session.

ments in performance not only occur during the training experience but rather could evolve after the termination of training was not until recently addressed. An animal model has provided evidence for a long-term consolidation phase in the auditory modality (Galvan and Weinberger 2002). In humans, Atienza et al. (2004) reported changes in the auditory event-related potentials (ERP) at $48-72 \mathrm{~h}$ post-training following a single training session on discriminating a complex auditory pattern. While participants showed a significant improvement during the training session, and ERP changes were found at $48 \mathrm{~h}$ post-training in the sleep allowed group, clear evidence for delayed gains in auditory performance was not demonstrated (Atienza et al. 2004). Our findings of robust delayed gains in the human auditory modality (Ari-Even Roth et al. 2003) are in agreement with those recently reported by Gaab et al. (2004) in a nonverbal task. However, in the latter study delayed gains occurred only when a night's sleep was allowed. In two recent studies (Fenn et al. 2003; Gottselig et al. 2004) no delayed gains in performance, compared with immediate post-training, were found even following sleep. Fenn et al. (2003) have found that time in the waking state may even degrade the gains in performance accrued during training on a language-related auditory task. A night's sleep, however, was effective in facilitating the recovery and subsequent recognition of the learned material following a single session of training.
In the present study, the gains in performance generalized partly but not fully to an untrained token pair or to performance with the untrained ear (results to be reported in detail elsewhere; see also Ari-Even Roth et al. 2004). This pattern of results supports the notion that a specific improvement in the identification of the trained stimuli has occurred rather than a nonspecific improvement in auditory discrimination.

While the study was not specifically designed to establish the mechanisms whereby time in sleep and time in the awake state contribute to the evolution of delayed gains in auditory skill learning, the results of our two experiments show that the evolution of delayed gains in performance did not necessarily depend on sleep. This result is independent of the fact that in experiment 2 diurnal (circadian rhythm) factors were not controlled for and may have contributed to the daytime (waking state) gains (e.g., Fischer et al. 2002). In some participants, additional gains were accrued also during the $12-24 \mathrm{~h}$ post-training interval (irrespective of whether sleep occurred in the interval), suggesting that the processes presumably subserving the evolution of delayed gains may still be active many hours after the termination of training (see also Hauptmann and Karni 2002; Korman et al. 2003; Walker et al. 2003). Thus, it is not clear whether sleep following the waking time consolidation interval may have further contributed to the long-term retention of the delayed gains.

As a recent review has pointed out, the necessity of sleep for effective consolidation is still under debate (Maquet et al. 2003a). While several studies on visual and motor skill learning reported delayed gains in performance evolving in the awake state, at least in some participants (Karni and Sagi 1993; Karni et al. 1994; Shadmehr and Holcomb 1997; Gais et al. 2000; Fischer et al. 2002; Maquet et al. 2003b), others, often using different versions of these tasks, found significant improvement only after a night's sleep (Stickgold et al. 2000b; Walker et al. 2002, 2003; Korman et al. 2003). Our results are in accordance with studies of visual discrimination learning (Karni and Sagi 1993; Karni et al. 1994; Gais et al. 2000), and in a visuo-motor task (Maquet et al. 2003b) reporting that, at least for some individuals, significant delayed gains in performance were accrued also in the awake state. The source for these inconsistent findings is not clear (Maquet et al. 2003a; Korman et al. 2005). First, the amount of interference afforded by the intervening daytime experience may be critical in determining the relative effectiveness of awake state consolidation (Brashers-Krug et al. 1996; Hauptmann and Karni 2002; Walker et al. 2003). An argument of this nature was suggested by Fenn et al. (2003) and Gottselig et al. (2004) to explain the deterioration in performance during active waking state but not when sleep was allowed. Second, the triggering, and perhaps the rate of evolution, of delayed gains in performance may depend on the amount of training afforded during the training experience (Karni 1996; Hauptmann and Karni 2002) and the training schedule (Korman et al. 2003) a factor uncontrolled for across studies. This factor may explain the large differences in delayed gains found after a night's sleep following a single session versus two training sessions in the Gaab et al. (2004) study. It was also suggested that when less than optimal training was given, two cycles rather than one sleep cycle were needed for delayed gains to occur (Hauptmann and Karni 2002). Third, it is clear that some training schedules may even result in significant transient losses in performance, which may be recovered by sleep (Mednick et al. 2002; Fenn et al. 2003). Fourth, the nature of the training material, specifically the variability between the actual contents (stimuli) presented in the training events, may be a critical factor in determining the time-course of learning (Ofen-Noy et al. 2003). It was previously suggested that the evolution of delayed gains in perceptual tasks may depend on factors such as keeping task difficulty above but near threshold, avoiding floor effects in 
the measurement of performance, and allowing sufficient training in each session (Karni and Bertini 1997). Robertson et al. (2004) recently reported that the dependence of the evolution of delayed gains in the performance of a motor sequence on sleep was determined by the nature of the instructions.

The time-course of the evolution of delayed gains, and their retention, in our auditory identification task is similar to the one reported by Karni and Sagi (1993) in their version of the visual texture target discrimination task. The demonstration that some training schedules can even effect transient, specific losses of performance during the awake state, recoverable by sleep, in both the visual and auditory modalities (Mednick et al. 2002; Fenn et al. 2003) provide another indication for the similarity between learning processes in the two sensory systems. Thus our results support the parsimonious notion that common processes may subserve memory consolidation during normal sleep and the awake state in the visual and auditory modalities, given similar task and training constraints (Karni et al. 1994; Karni 1996). We cannot rule out, however, that contrary to this parsimonious notion a process or mechanism available only during sleep (see Ribeiro et al. 1999; Korman et al. 2005) may be critical for the evolution of delayed gains in specific tasks or specific processing systems (see Dudai 1996).

\section{Materials and Methods}

\section{Subjects}

A total of 48 right-handed, native Hebrew-speaking, female subjects participated in the experiments. Their mean age was $23.41 \pm 2.46 \mathrm{yr}$ (range $=18-26 \mathrm{yr}$ ). All subjects had normal hearing sensitivity in both ears (pure-tone air-conduction thresholds $\leq 15 \mathrm{~dB}$ at octave frequencies of $250-8000 \mathrm{~Hz}$ ) (ANSI 1989). Participants had no history of hearing or speech disorders or of sleep-wake cycle disruptions. All were naive to the experimental procedure and had not participated in similar experiments before. Subjects were instructed not to take naps during daytime and to abstain from alcoholic beverages. To be included in experiment 2, at least $6 \mathrm{~h}$ of sleep were required on the target night. The study was approved by the Institutional Review Board of Tel-Aviv University. Informed consent was obtained from all subjects. Subjects were paid for their participation.

\section{Stimuli}

The stimuli in the present study consisted of naturally spoken stop CV syllables /da/-/ga/ embedded in noise (Fig. 4A-C). These specific syllables were chosen as they constitute a minimal pair differing only in the place of articulation. Thus, they are more susceptible to noise as they are highly dependent on spectral cues for perception (e.g., second formant transition) (Gelfand et al. 1986). The ability to discriminate such speech sounds in noise is an important factor in normal speech perception, and a deficiency in this ability characterizes children and adults with auditory processing disorders (see Jerger and Musiek 2000), hearing impairment (Beattie et al. 1997), and normal aging (Weinstein 2000). Two productions of each of the /da/ and /ga/ syllables were digitally recorded from a female native Hebrew speaker at a sampling rate of $22,050 \mathrm{~Hz}$ in $16 \mathrm{bits}$, normalized, and edited to a total duration of $300 \mathrm{msec}$. White Gaussian noise was gated with a 10 -msec cosine-raised rise-fall time. Each of the two versions of each token was then mixed with five different segments of noise, thus resulting in within-syllable variability due to the effect of different noise pairing. The total duration of the syllables in noise was $400 \mathrm{msec}$, with the syllable introduced 50 msec after the onset and terminating 50 msec before the termination of the noise. The CV syllables were kept at a constant intensity of $55 \mathrm{dBHL}$ and mixed with the noise using a root mean square function at different SNRs from $15 \mathrm{~dB}$ to $-3 \mathrm{~dB}$ by varying the level of the masking noise.
A

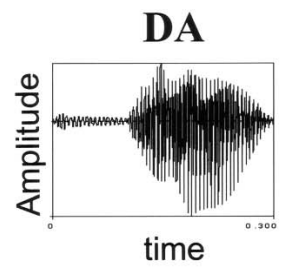

B

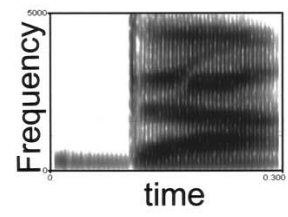

C

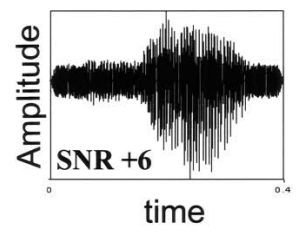

GA
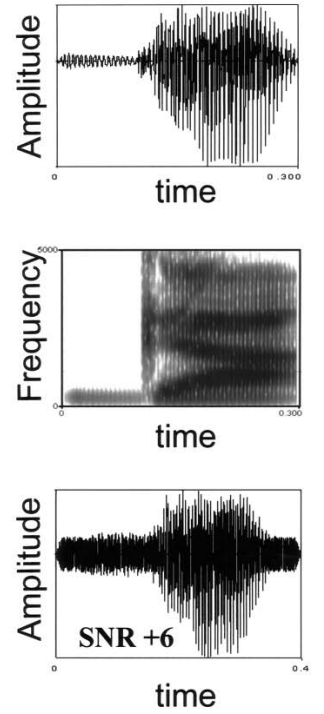

D

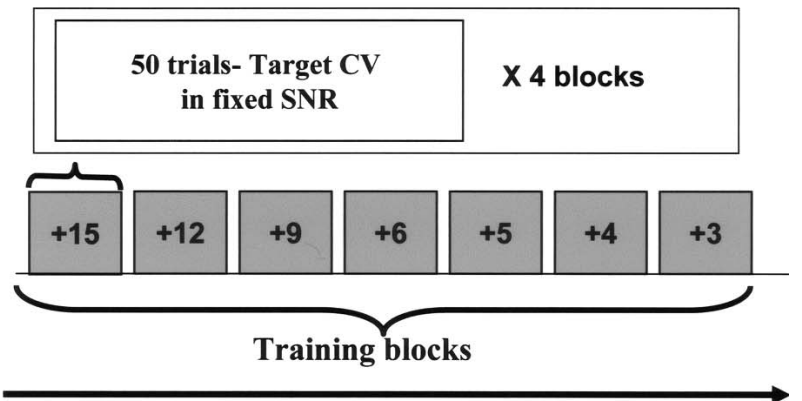

Decreasing SNR's

Figure 4. CV-syllables-in-noise identification task: stimuli and training paradigm. (A) Temporal envelopes of the trained syllables /da/ /ga/. (B) Temporal-spatial representations (spectrograms) of the stimuli. (C) Temporal envelopes of the syllables embedded in white Gaussian noise. (D) Training paradigm.

\section{Learning task}

A two-alternative forced choice design was used in a single, intensive, monaural training session. The session lasted 60-75 min and consisted of a total of 24-28 blocks (1200-1400 trials). Each training and testing block consisted of 50 target syllables embedded in noise at a constant SNR. Within each block, all five tokennoise segment pairs of each of the two target syllables were presented randomly in a balanced manner. Four successive blocks were presented at each SNR. Training started at $S N R=15 \mathrm{~dB}$, decreased in 3-dB or 1-dB steps depending on the subject's performance, and stopped at chance level performance (Fig. 4D). The post-training tests consisted of four to five blocks at an SNR corresponding to the one at which $75 \%-80 \%$ accuracy was achieved during the session. Visual feedback (trial by trial) was given only on the first two blocks. Stimuli were presented monaurally (Sennheiser HD 280 headphones). Responses were recorded by using Super Lab Pro system V 2.0 (Cedrus Corp.) with a two-button response box.

Each participant was randomly assigned to training on one of the token pairs (one /da/ and one /ga/; each paired with five different noise segments) and was then tested with the same pair. Statistical analysis revealed no significant differences in discriminatory performance or learning between the token pairs or the trained ear (right versus left).

\section{Learning \& Memory}




\section{Design and procedure}

\section{Experiment 1}

Participants trained in the morning ( 9 a.m. $\pm 1 \mathrm{~h}$ ). To test whether time-dependent gains in performance could evolve after the termination of the training session, the gains in performance were assessed at two time points following a single intensive training session: (1) on the day of training, within $1-12 \mathrm{~h}$ posttraining, and (2) at $24 \mathrm{~h}$ post-training (Fig. 1A). Twenty-two participants (mean age $=22.41 \pm 2.46 \mathrm{yr}$ ) participated in this experiment. Eleven participants were tested at $1-5 \mathrm{~h}$ post-training and 11 at $6-12 \mathrm{~h}$ post-training. All participants were retested at $24 \mathrm{~h}$ post-training, and 13 participants were also retested at $48 \mathrm{~h}$ post-training. To exclude the possibility that gains in performance observed $24 \mathrm{~h}$ post-training were influenced by the experience gained during the first post-training test (at $1-12 \mathrm{~h}$ posttraining), a control experiment was conducted with six additional subjects. Each subject was tested only once following training, at $24 \mathrm{~h}$ post-training.

To establish the robustness of retention, nine subjects were retested at $1 \mathrm{mo}$ and six others after an interval of 6 mo posttraining (Fig. 1A).

\section{Experiment 2}

In order to test for a possible differential effect on performance when normal sleep was afforded post-training, 20 additional subjects were randomly assigned to one of two subgroups: an overday group (10 participants, mean age $=22.99 \pm 1.55 \mathrm{yr}$ ) and an overnight group (10 participants, mean age $=24.14 \pm 1.58 \mathrm{yr}$ ). The over-day and overnight groups were trained at $8 \mathrm{a} . \mathrm{m}$. and 8 p.m., respectively, and both groups were retested at 12 and $24 \mathrm{~h}$ post-training (Fig. 1B). Participants were allowed normal everyday activity after the completion of the training session. Daytime napping was not allowed. Each participant agreed to allow for $>6$ $\mathrm{h}$ of sleep, to sleep in her normal abode, and to measure and report her sleep time. One participant in the overnight group reported $<6 \mathrm{~h}$ of sleep on the target night and was excluded. All participants reported normal sleep of $>6 \mathrm{~h}$ duration.

The ability to generalize the learning gains to untrained conditions (i.e., untrained /da/-/ga/ token pair produced by the same speaker, untrained ear) was tested following the $24 \mathrm{~h}$ posttraining test but is not reported in details in the current article (Ari-Even Roth et al. 2004).

\section{Data analysis}

Gains in performance were calculated as the increase in identification performance during each of the two post-training tests in relation to the performance in the training session, measured as percentage of correct and d' (a measure of discriminability based on the hit and false alarm rates). Four participants (two in experiment 1 and two in experiment 2) showed no significant improvement in any of the two post-training tests (including at 24 $\mathrm{h}$ post-training); these nonlearners were not included in the statistical analysis.

\section{Acknowledgments}

This work was partially supported by the Yairi Foundation. We thank E. Shabtai for statistical analysis and Eng. E. Sachratov for technical assistance.

\section{References}

American National Standards Institute (ANSI). 1989. Specifications for audiometers, ANSI S3.6. ANSI, New York.

Ari-Even Roth, D., Hildesheimer, M., Kishon-Rabin, L., and Karni, A. 2003. The beneficial effect of time after practice: Evidence for a consolidation process in auditory identification learning. Satellite symposium: "Plasticity of the central auditory system and processing of complex acoustic signals" International Brain Research Organization (IBRO) 67. . 2004. Does learning in a speech-in-noise identification task generalize to the untrained ear? J. Basic Clin. Physiol. Pharmacol. 15: 141 .

Atienza, M., Cantero, J.L., and Stickgold, R. 2004. Posttraining sleep enhances automaticity in perceptual discrimination. J. Cogn. Neurosci. 16: 53-64.
Beattie, R.C., Barr, T., and Roup, C. 1997. Normal and hearing-impaired word recognition scores for monosyllabic words in quiet and noise. Br. J. Audiol. 31: 153-164.

Brashers-Krug, T., Shadmehr, R., and Bizzi, E. 1996. Consolidation in human motor memory. Nature 382: 252-255.

Demany, L. 1985. Perceptual learning in frequency discrimination. J. Acoust. Soc. Am. 78: 1118-1120.

Dudai, Y. 1996. Consolidation: Fragility on the road to the engram. Neuron 17: 367-370.

Fenn, K.M., Nusbaum, H.C., and Margoliash, D. 2003. Consolidation during sleep of perceptual learning of spoken language. Nature 425: 614-616.

Fischer, S., Hallschmid, M., Elsner, A.L., and Born, J. 2002. Sleep forms memory for finger skills. Proc. Natl. Acad. Sci. 99: 11987-11991.

Gaab, N., Paetzold, M., Becker, M., Walker, M.P., and Schlaug, G. 2004. The influence of sleep on auditory learning: A behavioral study. Neuroreport 15: 731-734.

Gais, S., Plihal, W., Wagner, U., and Born, J. 2000. Early sleep triggers memory for early visual discrimination skills. Nat. Neurosci. 3: $1335-1339$.

Galvan, V.V. and Weinberger, N.M. 2002. Long-term consolidation and retention of learning-induced tuning plasticity in the auditory cortex of the guinea-pig. Neurobiol. Learn. Mem. 77: 78-108.

Gelfand, S.A., Piper, N., and Silman, S. 1986. Consonant recognition in quiet and in noise with aging among normal hearing listeners. $J$. Acoust. Soc. Am. 80: 1589-1598.

Gottselig, J.M., Hofer-Tinguely, G., Borbely, A.A., Regel, S.J., Landolt, H.P., Retey, J.V., and Achermann, P. 2004. Sleep and rest facilitate auditory learning. Neuroscience 127: 557-561.

Hauptmann, B. and Karni, A. 2002. From primed to learn: The saturation of repetition priming and the induction of long-term memory. Brain Res. Cogn. Brain Res. 13: 313-322.

Hikosaka, O., Nakahara, H., Rand, M.K., Sakai, K., Lu, X., Nakamura, K., Miyachi, S., and Doya, K. 1999. Parallel neural networks for learning sequential procedures. Trends Neurosci. 22: 464-471.

Jerger, J. and Musiek, F. 2000. Report of the consensus conference on the diagnosis of auditory processing disorders in school-aged children. J. Am. Acad. Audiol. 11: 467-474.

Karni, A. 1996. The acquisition of perceptual and motor skills: A memory system in the adult human cortex. Brain Res. Cogn. Brain Res. 5: 39-48.

Karni, A. and Bertini, G. 1997. Learning perceptual skills: Behavioral probes into adult cortical plasticity. Curr. Opin. Neurobiol. 7: $530-535$.

Karni, A. and Sagi, D. 1991. Where practice makes perfect in texture discrimination: Evidence for primary visual cortex plasticity. Proc. Natl. Acad. Sci. 88: 4966-4970.

. 1993. The time course of learning a visual skill. Nature 365: 250-252.

Karni, A., Tanne, D., Rubenstein, B.S., Askenasy, J.J., and Sagi, D. 1994. Dependence on REM sleep of overnight perceptual skill. Science 265: 679-682.

Korman, M., Raz, N., Flash, T., and Karni, A. 2003. Multiple shifts in the representation of a motor sequence during the acquisition of skilled performance. Proc. Natl. Acad. Sci. 100: 12492-12497.

Korman, M., Flash, T. and Karni, A. 2005. Synaptic and system consolidation may explain the complex relationship between resistance to interference and the emergence of delayed gains in newly-acquired procedural memories (commentary on Walker MA: A refined model of sleep and the time course of memory consolidation). Behav. Brain Sci. (in press).

Maquet, P., Laureys, S., Peigneux, P., Fuchs, S., Petiau, C., Phillips, C., Aerts, J., Del Fiore Degueldre, C., Meulemans, T., Luxen, A., et al. 2000. Experience-dependent changes in cerebral activation during human sleep. Nat. Neurosci. 3: 831-836.

Maquet, P., Laureys, S., Perrin, F., Ruby, P., Melchior, G., Boly, M., Vu, T.D., Desseilles, M., and Peigneux, P. 2003a. Festina lente: Evidences for fast and slow learning processes and a role for sleep in human motor skill learning. Learn. Mem. 10: 237-239.

Maquet, P., Schwartz, S., Passingham, R., and Frith, C. 2003b. Sleep-related consolidation of a visuomotor skill: Brain mechanisms as assessed by functional magnetic resonance imaging. J. Neurosci. 23: 1432-1440.

Mednick, S.C., Nakayama, K., Cantero, J.L., Atienza, M., Levin, A.A., Pathak, N., and Stickgold, R. 2002. The restorative effect of naps on perceptual deterioration. Nat. Neurosci. 5: 677-681.

Ofen-Noy, N., Dudai, Y., and Karni, A. 2003. Skill learning in mirror reading: How repetition determines acquisition. Brain Res. Cogn. Brain Res. 17: 507-521.

Peigneux, P., Laureys, S., Delbeuck, X., and Maquet, P. 2001. Sleeping brain, learning brain: The role of sleep for memory systems. Neuroreport 12: A111-A124. 
Ari-Even Roth et al.

Ribeiro, S., Goyal, V., Mello, C.V., and Pavlides, C. 1999. Brain gene expression during REM sleep depends on prior waking experience. Learn. Mem. 6: 500-508.

Robertson, E.M., Pascual-Leone, A., and Press, D.Z. 2004. Awareness modifies the skill-learning benefits of sleep. Curr. Biol. 14: 208-212.

Shadmehr, R. and Holcomb, H.H. 1997. Neural correlates of motor memory consolidation. Science 277: 821-825.

Stickgold, R. 1998. Sleep: Off-line memory reprocessing. Trends Cogn. Sci. 2: 484-492.

Stickgold, R., James, L., and Hobson, J.A. 2000a. Visual discrimination learning requires sleep after training. Nat. Neurosci. 3: 1237-1238.

Stickgold, R., Whidbee, D., Schirmer, B., Patel, V., and Hobson, J.A. 2000b. Visual discrimination task improvement: A multi-step process occurring during sleep. J. Cogn. Neurosci. 12: 246-254.

Stickgold, R., Fosse, R., and Walker, M.P. 2002. Linking brain and behavior in sleep-dependent learning and memory consolidation. Proc. Natl. Acad. Sci. 99: 16519-16521.
Tremblay, K., Kraus, N., and McGee, T. 1998. The time course of auditory perceptual learning: Neurophysiological changes during speech-sound training Neuroreport 9: 3557-3560.

Walker, M.P., Brakefield, T., Morgan, A., Hobson, J.A., and Stickgold, R. 2002. Practice with sleep makes perfect: Sleep-dependent motor skill learning. Neuron 35: 205-211.

Walker, M.P., Brakefield, T., Hobson, J.A., and Stickgold, R. 2003. Dissociable stages of human memory consolidation and reconsolidation. Nature 425: 616-620.

Watson, C.S. 1980. The time course of auditory perceptual learning. Ann. Otol. Rhino. Laryngol. Suppl. 89: 96-102.

Weinstein, B.E. 2000. Geriatric audiology. Thieme Medical Publishers, New York.

Received October 12, 2004; accepted in revised form January 11, 2004. 


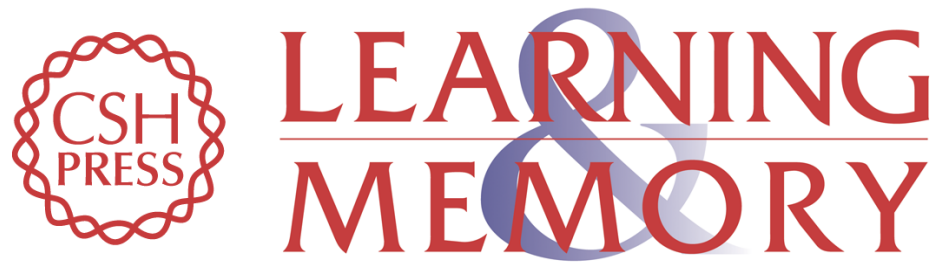

\section{A latent consolidation phase in auditory identification learning: Time in the awake state is sufficient}

Daphne Ari-Even Roth, Liat Kishon-Rabin, Minka Hildesheimer, et al.

Learn. Mem. 2005, 12:

Access the most recent version at doi:10.1101/87505

References This article cites 39 articles, 9 of which can be accessed free at:

http://learnmem.cshlp.org/content/12/2/159.full.html\#ref-list-1

License

Email Alerting Receive free email alerts when new articles cite this article - sign up in the box at the Service top right corner of the article or click here. 